\title{
Single photon nonlinear optics in photonic crystals
}

\author{
Dirk Englund, Ilya Fushman, Andrei Faraon, and Jelena Vučković \\ Ginzton Laboratory, Stanford University, Stanford, CA 94305
}

\begin{abstract}
We coherently probe a quantum dot that is strongly coupled to a photonic crystal nano-cavity by scattering of a resonant laser beam. The coupled system's response is highly nonlinear as the quantum dot saturates with nearly one photon per cavity lifetime. This system enables large amplitude and phase shifts of a signal beam via a control beam, both at single photon levels. We demonstrate photon-photon interactions with short pulses in a system that is promising for ultra-low power switches and two-qubit quantum gates.
\end{abstract}

\section{INTRODUCTION}

The photonic crystal-quantum dot system offers a scalable and robust platform for quantum optics research and the development of quantum information processing applications. In this approach, researchers aim to create a quantum network, which combines the utility of the photon as an information carrier with the nonlinearity of an atomic system for interacting more than one quantum bit (qubit) in a gate. The quantum network requires a way to coherently probe an atom or quantum dot in a cavity. Several proposals for scalable quantum information networks and quantum computation rely on direct probing of the cavity-quantum dot coupling by means of resonant light scattering from strongly or weakly coupled dots. ${ }^{1-6}$ Such experiments were performed in atomic systems ${ }^{7-9}$ and superconducting circuit QED systems. ${ }^{10}$

We probe a single InAs quantum dot (QD) that is strongly coupled to a photonic crystal (PC) cavity using a laser beam resonant with the quantum dot. The quantum dot strongly modifies the cavity transmission and reflection spectra. ${ }^{11} \mathrm{Cavity-}$ resonant photons are prohibited from passing through the cavity at the QD resonance, as was simultaneously reported in microposts by Srinivasan and Painter. ${ }^{12}$ As the QD-resonant probe laser approaches an intensity corresponding to one photon inside the cavity, the quantum-dot induced reflectivity feature disappears as the quantum dot saturates. This saturation represents a giant optical nonlinearity that enables large nonlinear phase and amplitude shifts of a signal photon by the presence of a control photon. ${ }^{11,13}$
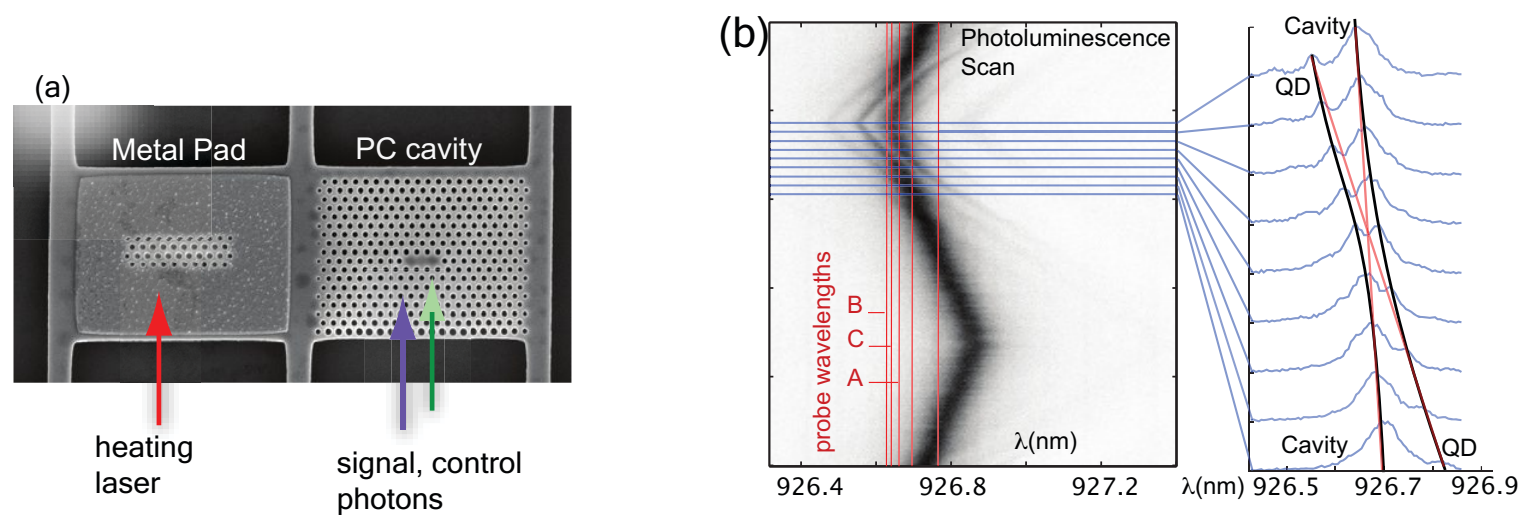

Figure 1. Resonant probing of QD/cavity system. (a) Photonic crystal structure containing cavity and laser heating pad. (b) Photoluminescence spectrum of strongly coupled QD/cavity system tuned through resonance by temperature tuning. ${ }^{14}$ (c) Reflected resonant signal beam as the cavity and quantum dot are scanned across the fixed probe by temperature tuning. The reflected intensity drops when the QD becomes resonant with the probe beam.

Advanced Optical Concepts in Quantum Computing, Memory, and Communication II, edited by Zameer U. Hasan, Alan E. Craig, Philip R. Hemmer, Proc. of SPIE Vol. 7225

72250F - @ 2009 SPIE · CCC code: 0277-786X/09/\$18 - doi: 10.1117/12.809305 

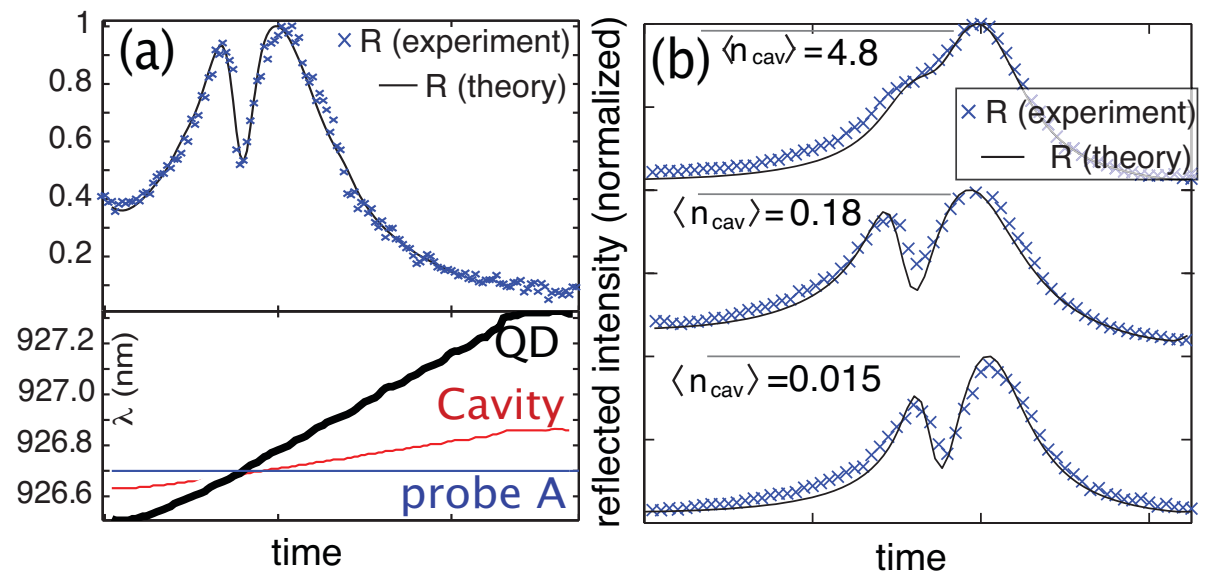

Figure 2. Resonant probing of QD/cavity system. (a) The cavity and quantum dot are scanned across the fixed probe by temperature tuning. The reflected intensity drops when the QD becomes resonant with the probe beam. (b) The QD is saturated at the single-photon level, representing a large optical nonlinearity. $\left\langle n_{\text {cav }}\right\rangle$ denotes the average photon number in the cavity.

\section{COHERENT OPTICAL DIPOLE ACCESS IN A CAVITY}

The structure consists of a linear three-hole defect cavity in a triangular photonic crystal lattice, as shown in Fig.1(a). It is fabricated in GaAs and contains a central layer of InAs quantum dots and has a quality factor $Q=10^{4}$. The temperature of the structure is scanned by a heating laser. ${ }^{14}$ The QD and cavity tune at different rates, as shown in the bottom panel of Fig.2(a). Fig.1(b) shows the photoluminescence spectra as the QD and cavity are tuned. They intersect in the characteristic anticrossing fashion of a strongly coupled system.

This system is then probed by reflecting a narrow-bandwidth laser beam that is near resonance with the QD and cavity. The reflectivity measurements are shown in Fig. 2(a). Instead a Lorentzian-shaped cavity spectrum, a drop in the reflected signal is observed at the QD wavelength, as expected from theory ${ }^{5}$ (fits).

We find good agreement between the measured reflectivity and theory, ${ }^{11}$ using the above-mentioned cavity/QD parameters and the tracked QD and cavity wavelengths shown in the bottom of Fig. 2(a). The QD-induced feature does not reach zero because of fluctuations in the heating power (and hence QD resonance), and because of 'blinking' of the QD exciton, probably by random charging. When these are taken into account in our fit by convolving it with a Gaussian filter (FWHM=0.005 nm), the theoretical model matches the data (black fits). Another reason why the dip does not reach closer to zero, as predicted by theory, is that the dot randomly jumps between different states which can be resonant or off-resonant from the cavity. The resonant state produces a dip while the off-resonant state does not. As a consequence, the dip height is averaged between the occupation probabilities. This 'blinking' was less significant than thermal jitter in this QD/cavity, but we have observed that it can play a big role in other systems. The reason for blinking is probably a combination of random charging ${ }^{15}$ and phonon-mediated jitter.

So far, we have shown reflectivity spectra obtained at the narrow wavelength range of the probe laser. This technique provides higher resolution than the spectrometer. However, if the quantum/cavity coupling is high, then the QD-induced feature can also be resolved when the cavity is probed with a broad-band source which is then analyzed on a spectrometer. For lack of a broad-band continuous-wave source near $928 \mathrm{~nm}$, we used the Ti:Sapph laser in pulsed mode ( $\sim 3$ ps duration and $\sim 0.3 \mathrm{~nm}$ width). The probe intensity was very low, about $1 \mathrm{nW}$ before the microscope's objective lens, so that the pulses do not saturate the quantum dot. The reflected signal is shown in Fig. 3(a), normalized by the roughly Gaussian spectrum of the probe. This QD/cavity system is identical in design to the one discussed above, with a $Q$ value near 10,000. The quantum dot has slightly higher coupling with $g / 2 \pi=16 \mathrm{GHz}$. Because of the limited resolution the spectrometer $(0.03 \mathrm{~nm})$, the features are blurred. Otherwise the reflected spectrum agrees with theory, which is plotted in Fig. 3(b). It is possible to probe the system in broad-band when its response is linear, as in this weak-excitation limit where the average intracavity photon number $\langle n\rangle \ll 1$. When the probe intensity grows, the two-level nature of the quantum dot causes a large nonlinearity. We discuss this next. 

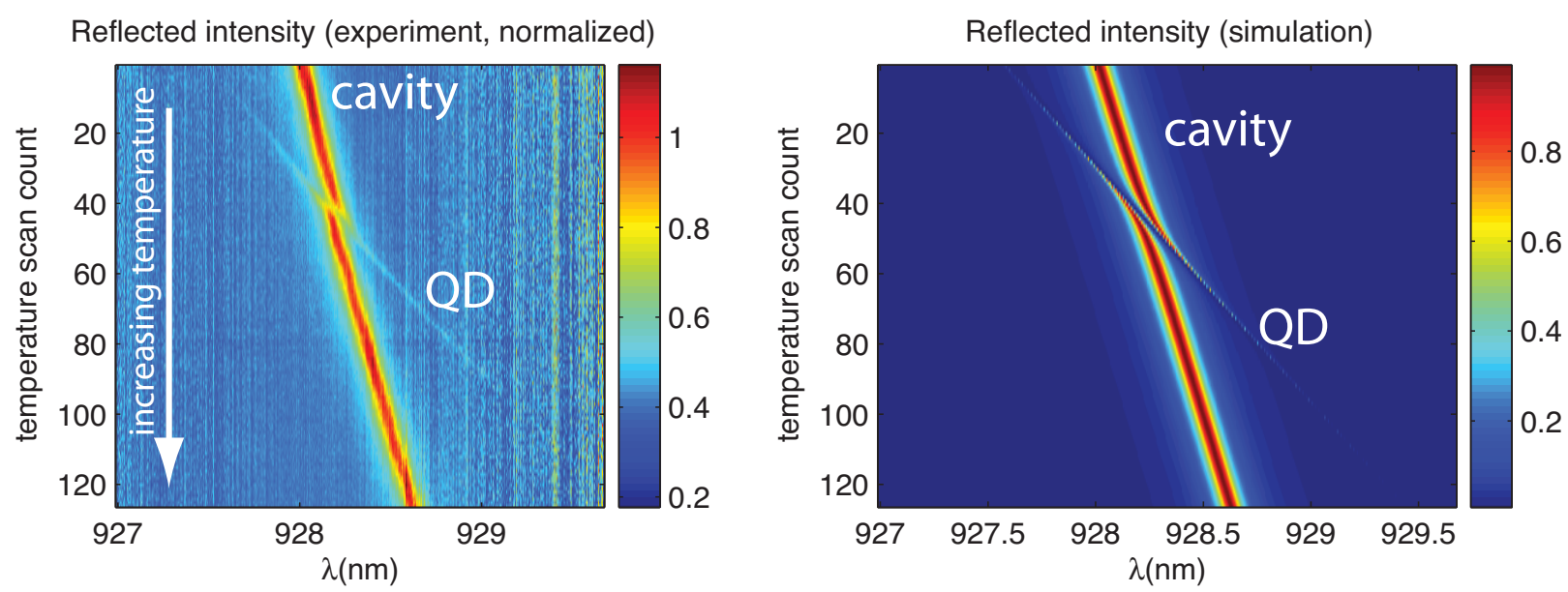

Figure 3. (a) Broad-band cavity reflectivity from cavity, as the quantum dot is tuned through the anticrossing point. (b) Reflected signal (theory).

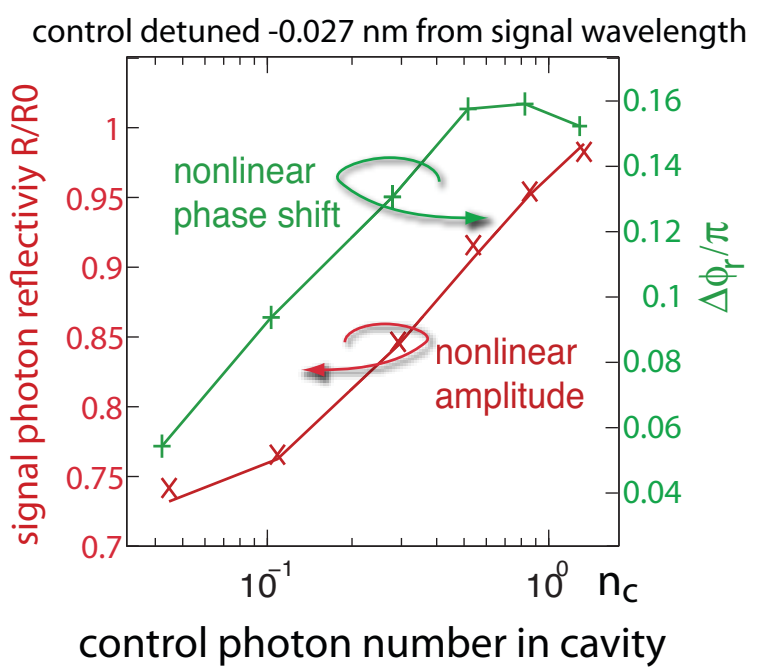

Figure 4. Saturation of the QD-induced interference and its corresponding phase. (a) The dot was detuned from the cavity by $g / 3.5$; the control and probe beams are identical here. The measured saturation agrees with theory (solid line). The dashed curves show the expected phase and intensity when the control intensity is doubled. The nonlinear phase shift $\phi_{r}\left(n_{c}\right)-\phi_{r}\left(2 n_{c}\right)$ is maximized at $n_{c}=0.1$, indicated by the arrow. (b) Nonlinear response to control photon number $n_{c}$ when the signal beam is $0.009 \mathrm{~nm}(\approx \mathrm{g} / 3)$ from the dot resonance (vertical lines in $\mathrm{a}, \mathrm{b})$.

\section{GIANT OPTICAL NONLINEARITY}

When the probe power is increased, we can explore the nonlinear behavior of the QD/cavity system. Fig.2(b) shows the QD-induced reflectivity dip vanishing as $P_{i n}$ is increased. The saturation sets in when there is approximately one photon coupled to the cavity per modified quantum dot lifetime, which corresponds to an average cavity photon population near one. We observe agreement with theory by a steady-state solution of the quantum master equation (solid lines). The QDinduced feature occurs through the destructive interference between the incident beam and that scattered by the photon, which is $\pi$ out of phase with it in reflection. This implies that the observed saturation of the QD-induced feature also entails a phase change in the reflected beam. Indeed, we observe a phase change of $0.24 \pi\left(43^{\circ}\right)$ when the control photon number is increased from $\langle n\rangle=0.08$ to $3 .^{13}$

We also explored the interaction of a signal beam and a detuned control beam. Detuned signal and control photons are useful for applications such as quantum quantum nondemolition (QND) detection, where the control beam accumulates a phase in the presence of the signal beam, or all-optical control, where a control beam switches the transmission of the cavity to the signal beam. For this measurement, we consider another dot that is also strongly coupled with a vacuum 
Rabi frequency $g / 2 \pi=8 \mathrm{GHz}$. We detuned the control beam by $\Delta \lambda=-0.027 \mathrm{~nm}(\simeq g)$ from the signal beam. With a constant signal intracavity number $n_{s} \approx 0.2$, we then varied the control photon number $n_{c}$. The photon-photon interaction is mediated primarily by the QD saturation through the control beam, though some detuning occurs by the AC Stark effect, which can create large phase shifts. ${ }^{16}$ In Fig. 4, we plot the phase and intensity of the signal beam when it is red-detuning by $0.4 g$ from the cavity, which in turn is resonant with the QD. Here the phase is plotted with respect to the phase when the control beam is off, $\Delta \phi_{r}=\phi_{r}-\phi_{r}\left(n_{c}=0\right) \equiv \phi_{r}-\phi_{r, 0}$.

The coherent QD access enabled by the CODAC technique is essential for quantum information processing in solidstate systems, as it opens the door to single photon detection, ${ }^{9}$ coherent transfer of the QD state to photon state, ${ }^{1}$ and quantum repeaters employing nondestructive Bell measurements with the addition of a third long lived QD level. ${ }^{5}$ The observed giant optical nonlinearity has promising applications for the generation of nonclassical and squeezed states of light, ${ }^{7,17}$ and non-demolition photon number state measurements. ${ }^{9,18}$ It may also find applications in classical all-optical signal processing. The photonic crystal approach can lower losses by reduced spontaneous emission ${ }^{19}$ and on-chip integration. ${ }^{20}$

\subsection{Acknowledgements}

Financial support was provided by the ONR Young Investigator Award and MURI Center for photonic quantum information systems (ARO/DTO Program). D.E. and I.F. were also supported by the NDSEG and NSF fellowships. Work was performed in part at the Stanford Nanofabrication Facility of NNIN supported by the National Science Foundation. We thank Nick Stoltz and Pierre Petroff of UC Santa Barbara for QD material.

\section{REFERENCES}

1. J. I. Cirac, P. Zoller, H. J. Kimble, and H. Mabuchi, "Quantum State Transfer and Entanglement Distribution among Distant Nodes in a Quantum Network," Phys. Rev. Lett. 78, pp. 3221-24, Apr. 1997.

2. A. Imamoğlu, D. D. Awschalom, G. Burkard, D. P. DiVincenzo, D. Loss, M. Sherwin, and A. Small, "Quantum Information Processing Using Quantum Dot Spins and Cavity QED," Phys. Rev. Lett. 83, pp. 4204-4207, Nov. 1999.

3. L.-M. Duan and H. J. Kimble, "Scalable photonic quantum computation through cavity-assisted interactions," Phys. Rev. Lett. 92(12), p. 127902, 2004.

4. L. Childress, J. M. Taylor, A. S. Sorensen, , and M. D. Lukin, "Fault-tolerant quantum repeaters with minimal physical resources and implementations based on single-photon emitters," Phys. Rev. A 72, p. 052330, 2005.

5. E. Waks and J. Vučković, "Dipole induced transparency in drop-filter cavity-waveguide systems," Phys. Rev. Lett. 96, April 2006.

6. T. D. Ladd, P. van Loock, K. Nemoto, W. J. Munro, and Y.Yamamoto, "Hybrid quantum repeater based on dispersive CQED interactions between matter qubits and bright coherent light," New Journal of Physics 8, p. 184, 2006.

7. K. M. Birnbaum, A. Boca, R. Miller, A. D. Boozer, T. E. Northup, and H. J. Kimble, "Photon blockade in an optical cavity with one trapped atom," Nature 436, pp. 87-90, 2005.

8. A. Rauschenbeutel, G. Nogues, S. Osnaghi, P. Bertet, M. Brune, J. M. Raimond, and S. Haroche, "Coherent Operation of a Tunable Quantum Phase Gate in Cavity QED," Phys. Rev. Lett. 83, pp. 5166-5169, 1999.

9. G. Nogues, A. Rauschenbeutel, S. Osnaghi, M. Brune, J. M. Raimond, and S. Haroche, "Seeing a single photon without destroying it," Nature 400, pp. 239-242, 1999.

10. D. I. Schuster, A. A. Houck, J. A. Schreier, A. Wallraff, J. M. Gambetta, A. Blais, L. Frunzio, J. Majer, B. Johnson, M. H. Devoret, S. M. Girvin, and R. J. Schoelkopf, "Resolving photon number states in a superconducting circuit," Nature 445, pp. 515-18, Feb 2007.

11. D. Englund, A. Faraon, I. Fushman, N. Stoltz, P. Petroff, and J. Vučković, "Controlling cavity reflectivity with a single quantum dot," Nature 450(6), pp. 857-61, 2007.

12. K. Srinivasan and O. Painter, "Linear and nonlinear optical spectroscopy of a strongly coupled microdisk-quantum dot system," Nature 450, pp. 862-865, Dec. 2007.

13. I. Fushman, D. Englund, A. Faraon, N. Stoltz, P. Petroff, and J. Vuckovic, "Controlled Phase Shifts with a Single Quantum Dot," Science 320(5877), pp. 769-772, 2008.

14. A. Faraon, D. Englund, I. Fushman, N. Stoltz, P. Petroff, and J. Vučković, "Local quantum dot tuning on photonic crystal chips," Appl. Phys. Lett. 90, May 2007. 
15. C. Santori, D. Fattal, J. Vučković, G. S. Solomon, E. Waks, and Y. Yamamoto, "Submicrosecond correlations in photoluminescence from inas quantum dots," Phys. Rev. B 69, p. 205324, May 2004.

16. E. Waks and J. Vuckovic, "Dispersive properties and large kerr nonlinearities using dipole-induced transparency in a single-sided cavity," Physical Review A (Atomic, Molecular, and Optical Physics) 73(4), p. 041803, 2006.

17. J. E. Reiner, W. P. Smith, L. A. Orozco, H. J. Carmichael, and P. R. Rice, "Time evolution and squeezing of the field amplitude in cavity QED,” J. Opt. Soc. Am. B 18, pp. 1911-21, 2001.

18. N. Imoto, H. Haus, and Y. Yamamoto, "Quantum nondemolition measurement of the photon number via the optical Kerr effect," Phys. Rev. A 32, pp. 2287-2292, 1985.

19. D. Englund, D. Fattal, E. Waks, G. Solomon, B. Zhang, T. Nakaoka, Y. Arakawa, Y. Yamamoto, and J. Vučković, "Controlling the Spontaneous Emission Rate of Single Quantum Dots in a Two-Dimensional Photonic Crystal," Phys. Rev. Lett. 95, p. 013904, July 2005.

20. D. Englund, A. Faraon, B. Zhang, Y. Yamamoto, and J. Vuckovic, "Generation and transfer of single photons on a photonic crystal chip," Opt. Express 15, pp. 5550-8, Apr. 2007. 